\title{
Brain-derived neurotrophic factor/tropomyosin-related kinase $B$ signaling pathway contributes to the aggressive behavior of lung squamous cell carcinoma
}

\author{
Keigo Ozono ${ }^{1,2}$, Yoshihiro Ohishi ${ }^{1}$, Hideya Onishi ${ }^{3}$, Katsuya Nakamura ${ }^{3,4}$, Junichi Motoshita ${ }^{5}$, Masato Kato ${ }^{6}$, \\ Ryoichi Nakanishi ${ }^{7}$, Masafumi Nakamura ${ }^{2}$ and Yoshinao Oda ${ }^{1}$
}

The tropomyosin-related kinase (Trk) family consists of TrkA, TrkB, and TrkC, which play essential roles in tumor progression and/or suppression in various cancers. Little is known about the biological significance of the Trk family in human lung squamous cell carcinoma (SCC). Here we investigated the clinical significance of the protein expression of Trk family members in samples from 99 SCC patients, and we explored the relationship between invasion/proliferation activities and Trk expression using lung SCC cell lines to clarify the biological significance of the Trk family in lung SCC. Immunohistochemical high expression of TrkB was significantly correlated with vascular invasion $(P=0.004)$, lymph node metastasis $(P<0.001)$, and advanced stage $(P=0.0015)$. The overall survival of the patients with TrkB-high expression was significantly shorter than those with TrkB-low expression $(P=0.0110)$. TrkA/TrkC expressions were not predictors of poor prognosis. An in vitro assay demonstrated that the inhibition of brain-derived neurotrophic factor (BDNF) (a TrkB ligand) and TrkB by K252a (a Trk inhibitor) or siRNA (BDNF-siRNA, TrkB-siRNA) suppressed the invasion, migration, and proliferative activities of lung SCC cells. The administration of recombinant human BDNF (rhBDNF) enhanced the invasion, migration, and proliferation activities, which were abrogated by K252a. TrkB-siRNA transfection increased the protein expression of E-cadherin and decreased vimentin expressions in lung SCC cells. Matrix metalloproteinase-2 (MMP-2)mediated gelatin degradations were decreased in lung SCC cells transfected with TrkB-siRNA. Thus, TrkB-high expression is an indicator of poor prognosis in lung SCC, probably due to invasion/proliferation activities promoted by the BDNF/TrkB signaling pathway, which could become a therapeutic target for lung SCC.

Laboratory Investigation (2017) 97, 1332-1342; doi:10.1038/labinvest.2017.45; published online 12 June 2017

Non-small-cell lung cancer (NSCLC) accounts for $\sim 80 \%$ of lung cancers and remains the most common cause of cancerrelated deaths worldwide. ${ }^{1}$ Squamous cell carcinoma (SCC) is the second most common subtype of NSCLC (adenocarcinoma is the first), accounting for $\sim 20-30 \%$ of NSCLC cases. $^{2}$ The treatment for a subset of lung adenocarcinomas has greatly improved due to the development of therapies using the epidermal growth factor receptor (EGFR) tyrosine kinase inhibitors gefitinib and erlotinib ${ }^{3-6}$ or an anaplastic lymphoma kinase (ALK) inhibitor, crizotinib.,

The efficacy of EGFR tyrosine kinase inhibitor (TKI) against lung SCC remains controversial, ${ }^{9-13}$ but an ALK TKI has been demonstrated to be effective, especially for a small subset of adenocarcinomas. ${ }^{7,8}$ Molecular target therapy for advanced SCC of the lung is not yet well established. The development of new molecular target therapies for advanced SCC of the lung is thus a pressing issue.

Members of the tropomyosin-related kinase (Trk) family are tyrosine kinase receptors: TrkA (NTRK1), TrkB (NTRK2), and TrkC (NTRK3). Primary ligands with high affinity are nerve growth factor (NGF) for TrkA, brainderived neurotrophic factor (BDNF) for TrkB, and neurotrophin-3 (NT-3) for TrkC. ${ }^{14,15}$ The Trk family regulates the development of the nervous system and neural origin cells, and Trk family members are involved in the maintenance of neural tissue. ${ }^{14-17}$

'Department of Anatomic Pathology, Kyushu University, Fukuoka, Japan; ${ }^{2}$ Department of Surgery and Oncology, Kyushu University, Fukuoka, Japan; ${ }^{3}$ Department of Cancer Therapy and Research, Graduate School of Medical Sciences, Kyushu University, Fukuoka, Japan; ${ }^{4}$ Department of Thoracic Surgery, Japan Community Health Care

Organization, Kyushu Hospital, Fukuoka, Japan; ${ }^{5}$ Department of Diagnostic Pathology, Hamanomachi Hospital, Fukuoka, Japan; ${ }^{6}$ Department of Surgery, Hiramatsu Hospital, Saga, Japan and ${ }^{7}$ Department of Oncology, Immunology and Surgery, Graduate School of Medical Sciences and Medical School, Nagoya City University, Nagoya, Japan Correspondence: Professor Y Oda, PhD, Department of Anatomic Pathology, Graduate School of Medical Sciences, Kyushu University, Maidashi 3-1-1, Higashi-ku, Fukuoka 812-8582, Japan.

E-mail: oda@surgpath.med.kyushu-u.ac.jp

Received 25 September 2016; revised 14 March 2017; accepted 21 March 2017 
Many studies have indicated that Trk family members also have oncogenic functions in several malignant tumors. ${ }^{18-44}$ Interestingly, the oncological roles of TrkA, TrkB, and TrkC are different among tumor types despite their close homology. For example, TrkA overexpression is associated with poor prognosis in thyroid carcinoma, ${ }^{18}$ breast cancer, ${ }^{19}$ and ovarian serous carcinoma, ${ }^{20}$ whereas TrkA acts as a tumor suppressor in neuroblastoma ${ }^{21}$ and colon cancer ${ }^{22}$ as shown in in vitro assays. TrkB overexpression was reported to act as an oncogenic molecule in pancreas cancer, ${ }^{23}$ hepatocellular cancer, ${ }^{24}$ prostate cancer, ${ }^{25}$ ovarian cancer, ${ }^{26}$ Wilm's tumor, ${ }^{27}$ gastric cancer, ${ }^{28,29}$ breast cancer, ${ }^{30}$ colon cancer, ${ }^{31-33}$ oral squamous cell carcinoma, ${ }^{34}$ head and neck squamous cell carcinoma, ${ }^{35}$ and neuroblastoma. ${ }^{36} \mathrm{TrkC}$ overexpression is reported as an indicator of good prognosis for medulloblastoma ${ }^{37}$ and colon cancer. ${ }^{38}$

With regard to lung cancer, Ricci et al. reported constant BDNF and TrkB expressions in human lung SCC samples. ${ }^{39}$ Higher TrkB expression in NSCLC including SCC was reported to be correlated with lymph node metastasis ${ }^{40}$ and vascular invasion. ${ }^{41}$ Okamura et al. reported that high $\operatorname{TrkB}$ expression was correlated with short periods of disease-free and overall survival in NSCLC patients. ${ }^{41}$ In addition, Terry et al. reported that positive TrkB staining was correlated with improved disease-specific survival and overall survival in lung SCC. ${ }^{42}$

In terms of the function of the BDNF/TrkB signaling pathway, it was reported that enhancement of the BDNF/ TrkB pathway induced invasion activity in pulmonary large cell neuroendocrine carcinoma (LCNEC) cells $^{43}$ and lung adenocarcinoma cells. ${ }^{40}$ The Trk inhibitor K252a was reported to inhibit the invasion activity of LCNEC cells, ${ }^{43}$ induce cell death ${ }^{44}$ and diminish the colony formation of adenocarcinoma cells. ${ }^{44}$ However, a functional analysis of the $\mathrm{BDNF} / \mathrm{TrkB}$ signaling pathway of lung SCC has not been established.

In this study, we hypothesized that BDNF/TrkB signal promotes proliferating, migratory and invasive phenotypes and cellular plasticity (eg, epithelial-mesenchymal transition [EMT] and MMP level) in lung SCC (Figure 1). Accordingly, we investigated ${ }^{43}$ the clinical significance of the protein expression of Trk family members using clinical samples of lung SCC, and we examined the relationship between the $\mathrm{BDNF} / \mathrm{TrkB}$ signaling pathway and the invasion/migration and proliferation activities of lung SCC cells.

\section{MATERIALS AND METHODS}

\section{Patients and Case Selection}

We retrospectively analyzed the cases of 99 patients with lung SCC who underwent curative surgical resection at the Department of Surgery and Oncology, Kyushu University Hospital, the Department of Surgery, Hamanomachi Hospital, and the Department of Thoracic Surgery, Shinkokura Hospital between January 1999 and January 2005 (Table 1).

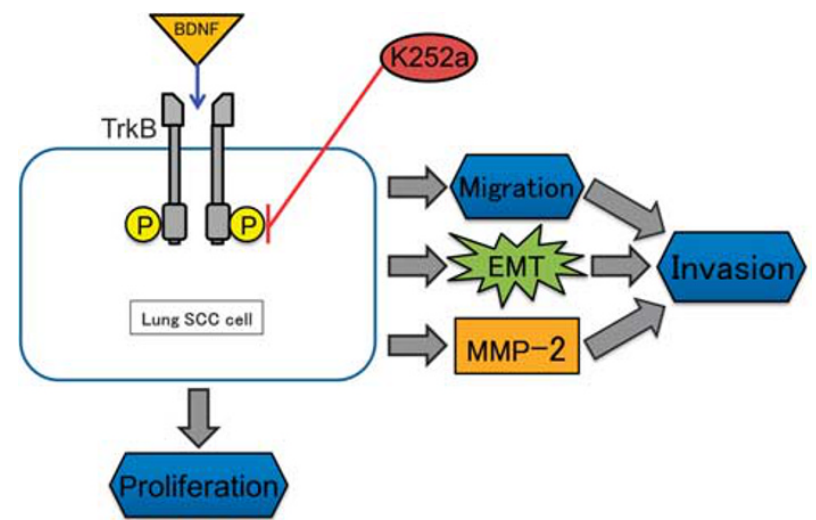

Figure 1 The role of the BDNF/TrkB signaling pathway in lung SCC. The BDNF/TrkB signaling pathway enhances the cell proliferation and invasion activity via migration, the EMT and MMP-2 activation.

All of the surgical specimens were routinely fixed by formalin before the histopathological and immunohistochemical evaluations. The patients' clinicopathological profiles are shown in Table 1. The histological subtypes of the tumors and the pathological stage were classified according to the World Health Organization (WHO) classification of lung and pleural tumors, ${ }^{45}$ and the Union for International Cancer Control (UICC) guidelines for tumor, node, metastasis (TNM) classification. ${ }^{46}$

This study was approved by the Ethics Committee of Kyushu University (No. 28-71).

\section{Immunohistochemistry}

Immunohistochemical staining (IHC) was performed using $4-\mu \mathrm{m}$-thick formalin-fixed, paraffin-embedded tissue sections and the primary antibodies TrkA (sc-118, Santa Cruz Biotechnology, Santa Cruz, CA, USA, 1:50), TrkB (sc-8316, Santa Cruz Biotechnology, 1:50) and TrkC (ab77494, Abcam, Cambridge, UK, 1:200). Endogenous peroxidase activity was blocked for $30 \mathrm{~min}$ by methanol containing $0.3 \%$ hydrogen peroxidase. For TrkA and TrkB, sections were incubated with primary antibodies overnight at $4{ }^{\circ} \mathrm{C}$, followed by a 40 -min incubation with the secondary antibodies at room temperature. For TrkC, antigen retrieval was conducted by microwave heating for $20 \mathrm{~min}$ with target retrieval solution (TRS) buffer. TrkC antibody incubation was followed by standard signal amplification, including rabbit LINKER (K8019) at room temperature for $15 \mathrm{~min}$, and then horseradish peroxidase (HRP)-conjugated EnVision FLEX (Dako, Glostrup, Denmark) at room temperature for $20 \mathrm{~min}$. The reaction products were visualized by diaminobenzidine (DAB).

We evaluated the proportion and intensity of the immunoreactive cells on the entire slides by using the Allred score (AS) as described. ${ }^{32}$ The proportional score (PS) was defined as follows: $0 \%=$ PS0, $0 \%<$ PS1 $\leq 1 \%$, $1 \%<$ PS2 $\leq 10 \%, 10 \%<$ PS3 $\leq 33 \%, 33 \%<$ PS4 $\leq 67 \%$, and $67 \%<$ PS5 $\leq 100 \%$. The intensity score (IS) was defined 
Table 1 Clinicopathological features and immunohistochemical TrkA, TrkB and TrkC expressions of patients with lung squamous cell carcinoma

\begin{tabular}{|c|c|c|c|c|c|c|c|c|c|c|}
\hline \multirow[b]{2}{*}{ Variable } & \multirow[b]{2}{*}{ Total } & \multicolumn{3}{|c|}{ TrkA } & \multicolumn{3}{|c|}{ TrkB } & \multicolumn{3}{|c|}{ TrkC } \\
\hline & & High $(n=33)$ & Low $(n=66)$ & $P$-value & High $(n=43)$ & Low $(n=56)$ & P-value & High $(n=19)$ & Low $(n=80)$ & $P$-value \\
\hline Age & $71.3(48-85)$ & $72.8(54-84)$ & $70.5(48-85)$ & & $71.3(55-84)$ & $71.3(48-85)$ & & $69.2(48-85)$ & 71.7 (54-85) & \\
\hline Sex & & & & 0.6833 & & & 0.5927 & & & 0.2166 \\
\hline Male & 85 & 29 & 56 & & 36 & 49 & & 18 & 67 & \\
\hline Grade & & & & 0.1471 & & & 0.6542 & & & 0.9454 \\
\hline $\mathrm{G} 1+\mathrm{G} 2$ & 30 & 13 & 17 & & 14 & 16 & & 6 & 24 & \\
\hline G3 & 67 & 19 & 48 & & 28 & 39 & & 13 & 54 & \\
\hline Vascular invasion & 26 & 9 & 17 & 0.9422 & 18 & 7 & $0.004^{*}$ & 2 & 24 & 0.0809 \\
\hline UICC T category & & & & 0.3939 & & & 0.3939 & & & 0.3614 \\
\hline $1+2$ & 88 & 29 & 59 & & 38 & 50 & & 16 & 72 & \\
\hline $3+4$ & 6 & 3 & 3 & & 3 & 3 & & 2 & 4 & \\
\hline UICC Stage & & & & 0.7561 & & & $0.0155^{*}$ & & & 0.1134 \\
\hline $1+\|$ & 83 & 29 & 54 & & 33 & 50 & & 17 & 66 & \\
\hline III & 10 & 3 & 7 & & 8 & 2 & & 0 & 10 & \\
\hline
\end{tabular}

Fisher's exact test was used to evaluate the associations between clinicopathological findings and biomarkers. ${ }^{*} P<0.05$.

as follows: IS0 $=$ negative, IS1 $=$ weak, IS2 $=$ intermediate, and IS3 $=$ strong.

The PS and IS were added to obtained a total score (TS) $(0$, 2 to 8 ), and divided into four grades: Grade 0 , a TS of 0; Grade 1, a TS of 2-4; Grade 2, a TS of 5 or 6, and Grade 3, a TS of 7 or 8 . Grades 2 and 3 were regarded as high expression.

\section{Cell Lines}

We purchased the human lung SCC cell lines LK-2, EBC-1, and RERF-LC-AI from Riken Bioresource Center (Tsukuba, Japan) and LC-1sq from the JCRB Cell Bank (Tokyo). The EBC-1 and RERF-LC-AI cells were cultured in MEM (Invitrogen-Gibco, Carlsbad, CA, USA) supplemented with $10 \%$ fetal bovine serum (FBS), $100 \mathrm{U} / \mathrm{ml}$ penicillin and $100 \mathrm{U} / \mathrm{ml}$ streptomycin. The LK-2 cells were cultured in RPMI 1640 (Invitrogen-Gibco) supplemented with 10\% FBS, $100 \mathrm{U} / \mathrm{ml}$ penicillin and $100 \mathrm{U} / \mathrm{ml}$ streptomycin. The LC-1sq cells were cultured with a 1:1 mixture of RPMI 1640 and Ham's F12 (Invitrogen-Gibco) supplemented with 10\% FBS, $100 \mathrm{U} / \mathrm{ml}$ penicillin and $100 \mathrm{U} / \mathrm{ml}$ streptomycin.

\section{Small Interfering RNA Transfection}

Small interfering RNA (siRNA) for TrkB (ON-TARGET plus SMART pool, L-003160), siRNA for BDNF (ON-TARGET plus SMART pool, L-017626), and negative control-siRNA
(ON-TARGET plus siCONTROL non-targeting pool, D-001810) were purchased from Dharmacon RNA Technologies (Chicago, IL, USA). Cells $\left(0.2 \times 10^{6}\right.$ cells per well) seeded in six-well plates were transfected with $100 \mathrm{nM}$ siRNA with the use of Lipofectamine RNAiMAX Reagent (Invitrogen, Carlsbad, CA, USA) according to the manufacturer's instructions. Target gene downregulation was confirmed after $48 \mathrm{~h}$.

\section{Western Blot Analysis}

Proteins were extracted with the use of PRO-PREP Protein Extraction Solution (iNtRON Biotechnology, Sungnam, Korea) and transferred to nitrocellulose membranes. The membranes were incubated with primary antibodies for TrkB (1:250), BDNF (sc-546, Santa Cruz Biotechnology, 1:250), GAPDH (sc-32233, Santa Cruz Biotechnology, 1:500), MMP-2 (sc-10736, Santa Cruz Biotechnology, 1:200), MMP-9 (sc-6840, Santa Cruz Biotechnology, 1:200), E-cadherin (clone-36/E-Cadherin, BD Transduction Laboratories, Franklin Lakes, NJ, USA, 1:2500) and vimentin (clone-V9, Dako, $1: 200)$ at $4{ }^{\circ} \mathrm{C}$ overnight, followed by peroxidase-labeled secondary antibodies at $37^{\circ} \mathrm{C}$. Immunoblots were identified using the ECL prime western blotting detection system (GE Healthcare Life Science, Buckinghamshire, UK) with a 
molecular imager (FX, Bio-Rad Laboratories, Hercules, CA, USA).

\section{Cell Invasion Assay and Migration Assay}

The cell invasion and migration assays were performed using the Trk inhibitor K252a (Alomone Labs, Jerusalem, Israel), siRNA (Control-siRNA, TrkB-siRNA, and BDNF-siRNA) and rhBDNF (Peprotech, Rocky Hill, NJ). The results of the invasion assay were assessed using a Biocoat Matrigel invasion chamber (BD Biosciences, San Diego, CA, USA) according to the manufacturer's protocol. In brief, the cells of each type were detached from the culture plate and resuspended in the Matrigel-coated upper chamber separated by an $8-\mu \mathrm{m}$ poresize filter at $1 \times 10^{5}$ cells per chamber in serum-free media. The outer wells were filled with media containing 5\% FBS.

After a 24 -h incubation at $37^{\circ} \mathrm{C}$ with $5 \%$ carbon dioxide, non-invading cells were wiped and removed using a cotton swab. Invading cells were fixed to the lower surface of the Transwell membrane with $70 \%$ ethanol and stained with hematoxylin-eosin. The membrane was cut and mounted on a microscope slide, and invading cells were counted in five random high-power fields.

The results of the migration assay were assessed using Falcon Cell Culture Inserts (Fisher Scientific, Pittsburgh, PA, USA). Cells of each type were detached and resuspended in the upper chamber separated by an $8-\mu \mathrm{m}$ pore-size filter at $1 \times 10^{5}$ cells per chamber in serum-free media. The bottom of each pore contained a polyethylene terephthalate (PET) membrane. The cells were incubated for $16 \mathrm{~h}$ at $37^{\circ} \mathrm{C}$ with $5 \%$ carbon dioxide. After that, non-migrating cells were wiped and removed. Migrating cells were fixed to the lower surface of the membrane with $70 \%$ ethanol and stained with hematoxylin-eosin. The membrane was cut and mounted on a microscope slide, and migrating cells were counted in five random high-power fields.

\section{Anchorage-Dependent Cell Proliferation Assay}

An anchorage-dependent cell proliferation assay was performed by using K252a, siRNA (Control-siRNA, TrkBsiRNA, and BDNF-siRNA) and rhBDNF as follows. In brief, each cell line $\left(2 \times 10^{3}\right.$ cells per well $)$ was plated in 96-well plates and cultured in medium containing 5\% FBS combined with the drugs described above for $48 \mathrm{~h}$. At regular intervals $(0,24$, and $48 \mathrm{~h})$, we measured the optical density at $492 \mathrm{~nm}$ (reference $620 \mathrm{~nm}$ ) using Cell Count Reagent SF (Nacalai Tesque, Kyoto, Japan). The ability of cell proliferation ability is expressed as the absorbance, reflecting the increased number of cells. The experiments were performed three times in triplicate wells.

\section{Gelatin Zymography}

We performed zymography by using a Gelatin zymography kit (Primary Cell, Ishikari, Japan), following the manufacturer's protocol. In brief, LK-2 and EBC- 1 cells $\left(0.2 \times 10^{6}\right.$ cells per well) seeded in six-well plates were transfected with
$100 \mathrm{nM}$ control-siRNA and TrkB-siRNA, or they were administered $10 \mathrm{ng} / \mathrm{ml} \mathrm{rhBDNF}$ and incubated for $24 \mathrm{~h}$ at $37^{\circ} \mathrm{C}$. The next day, the culture media were replaced with serum-free media and the cells were incubated again at $37^{\circ} \mathrm{C}$ overnight. After that, each medium was condensed and loaded for electrophoresis. The gel was washed and incubated for $24 \mathrm{~h}$ at $37^{\circ} \mathrm{C}$. The gel was bleached and scanned after being stained by stain solution. Areas of gelatin degraded by MMP were visualized as a clear zone against a blue background.

\section{Statistical Analysis}

All calculations were performed using JMP Statistical Discovery Software (ver. 11.0; SAS, Cary, NC, USA). All the data are presented as the mean \pm s.d. We used Fisher's exact test to evaluate the associations between the patients' clinicopathological findings and the biomarkers. We calculated survival curves using the Kaplan-Meier method, and compared the differences using the log-rank test. $P$-values $<0.05$ were considered significant.

\section{RESULTS}

Trk Family Member Expressions in Lung SCC Samples

The immunohistochemical expressions of TrkA, TrkB, and TrkC in the 99 cases of lung SCC and representative images are summarized and presented in Table 1 and Figure 2. Among the 99 tumor samples, a high expression of TrkA, TrkB, and TrkC was observed in 33 cases (33\%), 43 cases $(43 \%)$, and 19 cases (19\%), respectively.

There were no significant correlations between TrkA and the clinicopathological factors.

High TrkB expression was significantly correlated with vascular invasion $(P=0.004)$ and lymph node metastasis $(P<0.001)$. The advanced-stage cases showed significantly higher expressions of TrkB compared to the early-stage cases $(P=0.0015)$.

All of the advanced-stage cases showed low TrkC expression $(P=0.1134)$, but there were no significant correlations between TrkC expression and any of the clinicopathological factors (Table 1).

Information was available about the survival periods of all but one of the 99 lung SCC cases. There was no significant correlation between TrkA expression and survival $(P=0.1552)$ (Figure 2e).

The patients with high TrkB expression (TrkB-high) had significantly shorter survival periods compared to the patients with TrkB-low expression $(P=0.0110)$ (Figure 2f). The TrkChigh expression patients showed significantly longer survival periods compared to the patients with TrkC-low expression $(P=0.0289)$ (Figure $2 \mathrm{~g}$ ).

\section{Western Blotting Revealed Endogenous BDNF/TrkB Protein Expression in Two Cell Lines}

We examined the role of the BDNF/TrkB signaling pathway in lung SCC cells in an attempt to substantiate the clinical 

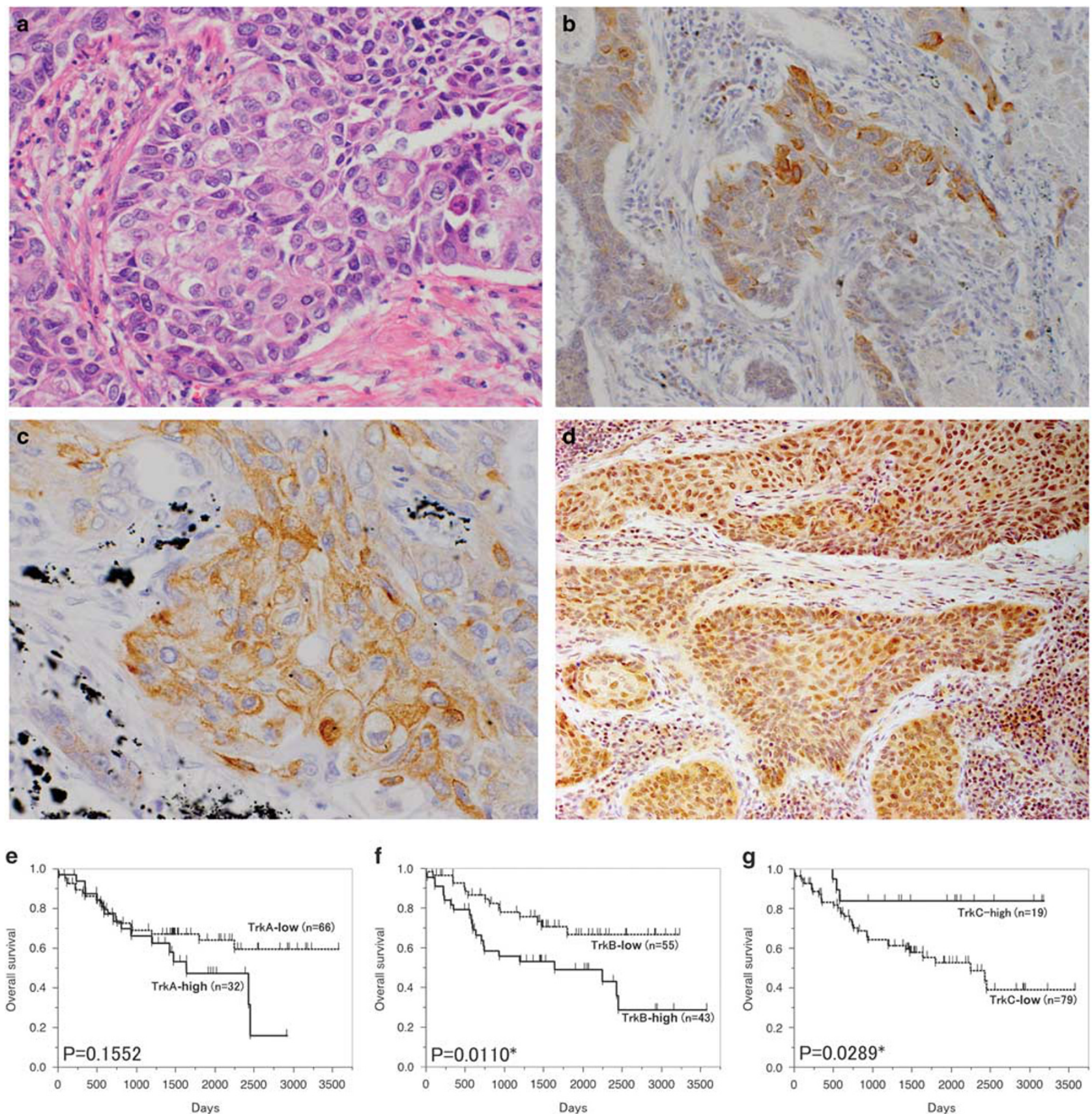

Figure 2 Histologic features and Trk immune-expressions in samples of lung SCC. (a) Hematoxylin and eosin staining of a representative lung SCC sample $(200 \times)$. (b) Immunohistochemical high expressions of TrkA, (c) TrkB, and (d) TrkC in tumor cells (200 x). Membranous and/or cytoplasmic staining is visible. Aberrant nuclear staining of TrkC is visible. Kaplan-Meier survival curves for SCC of lung patients with Trk-high and Trk-low tumors. (e) Patients with TrkB-high expression showed shorter overall survival compared to those with TrkB-low expression. (f) The TrkC-high patients showed longer survival compared to the TrkC-low patients. (g) There was no significant difference in overall survival between the TrkA-high and TrkA-low patients. $P$-values calculated with log-rank test are indicated. ${ }^{*} P<0.05$.

significance of TrkB expression demonstrated by immunohistochemical staining. We performed a western blotting analysis to reveal the protein expressions of endogenous TrkB and its ligand BDNF in four human lung SCC cell lines. Two of the four cell lines, ie, LK-2 and EBC-1, showed endogenous expression of both TrkB and BDNF (Figure 3a).

\section{The Inhibition of the BDNF/TrkB Signaling by the Trk Inhibitor K252a Suppressed the Invasion and Migration Phenotype of Lung SCC Cells}

To assess the effects of the BDNF/TrkB signaling pathway in lung SCC cells, we performed an invasion assay and a migration assay. The LK-2 and EBC-1 cells treated with 50 or 


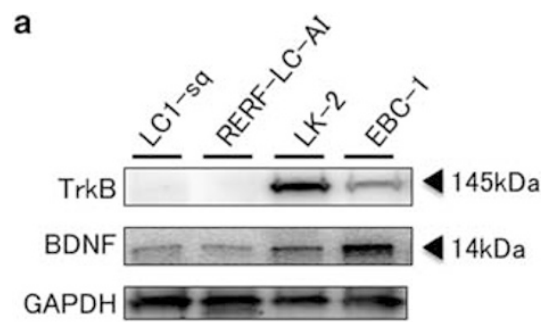

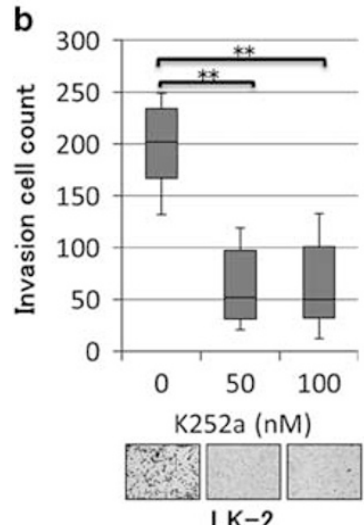

LK-2

d

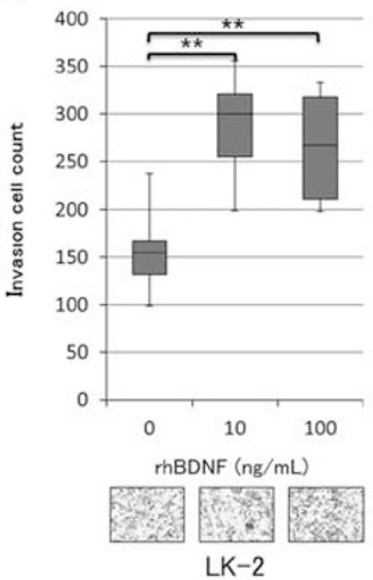

$\mathbf{f}$

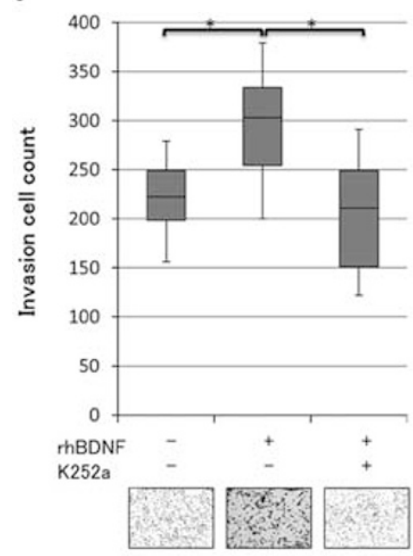

LK-2

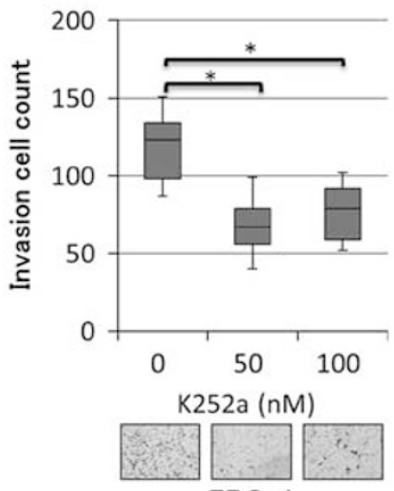

EBC-1
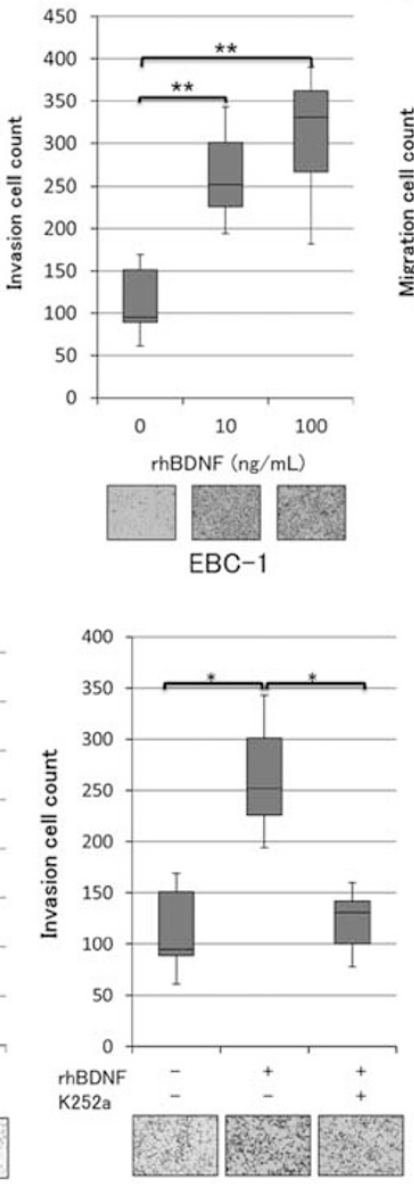

$\mathrm{EBC}-1$

e

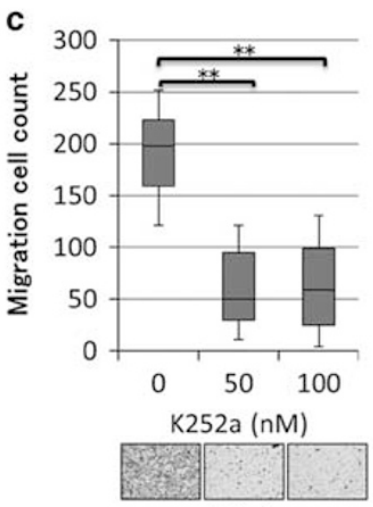

LK-2
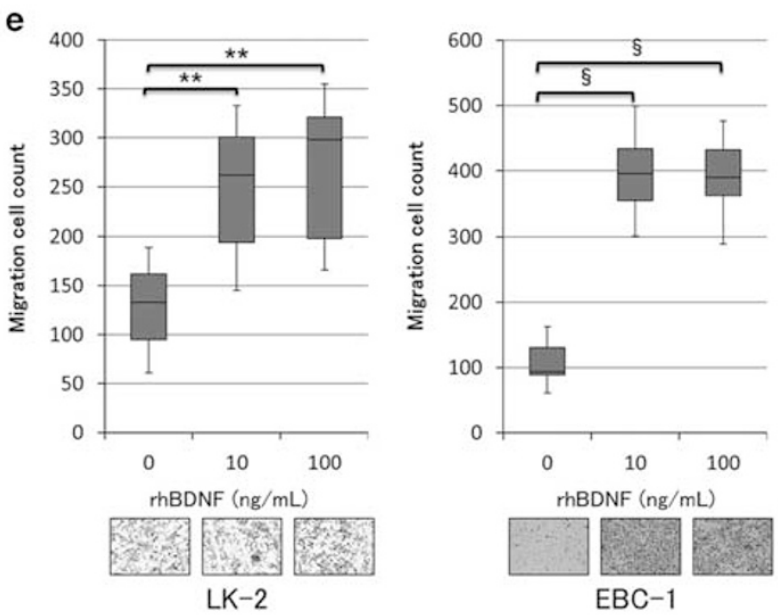

g

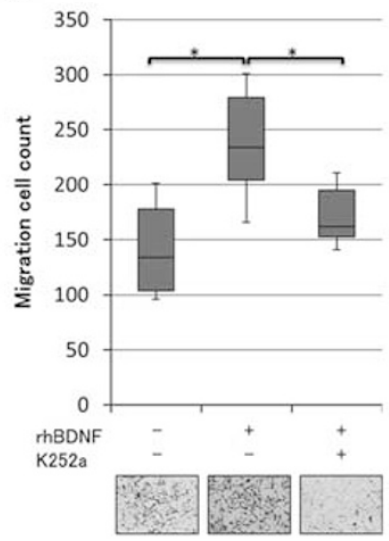

LK-2

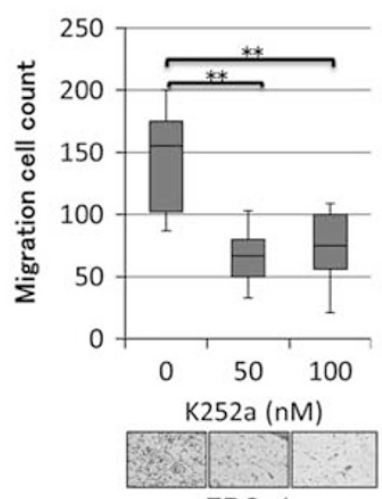

EBC-1

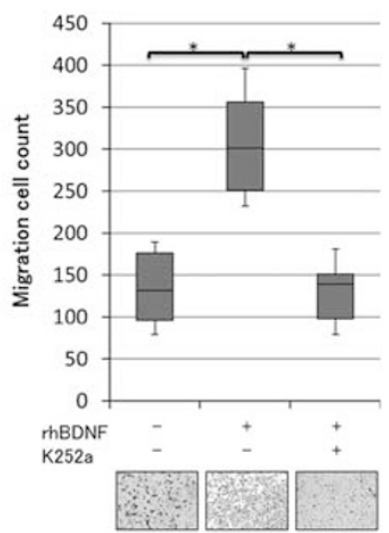

$\mathrm{EBC}-1$

Figure 3 For caption see page on 1338. 
$100 \mathrm{nM}$ K252a, a Trk inhibitor, showed significantly decreased numbers of invading cells and migrating cells compared to untreated cells (Figure $3 \mathrm{~b}$ and c). The RERF-LCAI cell, without endogenous TrkB expression, showed no significant changes in the numbers of invasion and migration cells irrespective of being treated with $50 \mathrm{nM} \mathrm{K252a}$ (Supplementary Figure S1).

\section{The Western Blot Analysis Confirmed the Downregulation of BDNF and TrkB by siRNA}

Western blotting confirmed the downregulation of TrkB and BDNF proteins extracted from the cells transfected with
TrkB-siRNA and BDNF-siRNA, respectively (Supplementary Figure S2A and B).

\section{The Inhibition of BDNF/TrkB Signaling by siRNA Suppressed the Invasion and Migration Phenotype of Lung SCC Cells}

The LK-2 and EBC-1 cells transfected with TrkB-siRNA or BDNF-siRNA showed significantly decreased numbers of invading cells and migrating cells (Supplementary Figure S2C, $\mathrm{D}$ respectively) compared to the cells transfected with Control-siRNA. The RERF-LC-AI cell, without endogenous TrkB expression, showed no significant changes in the
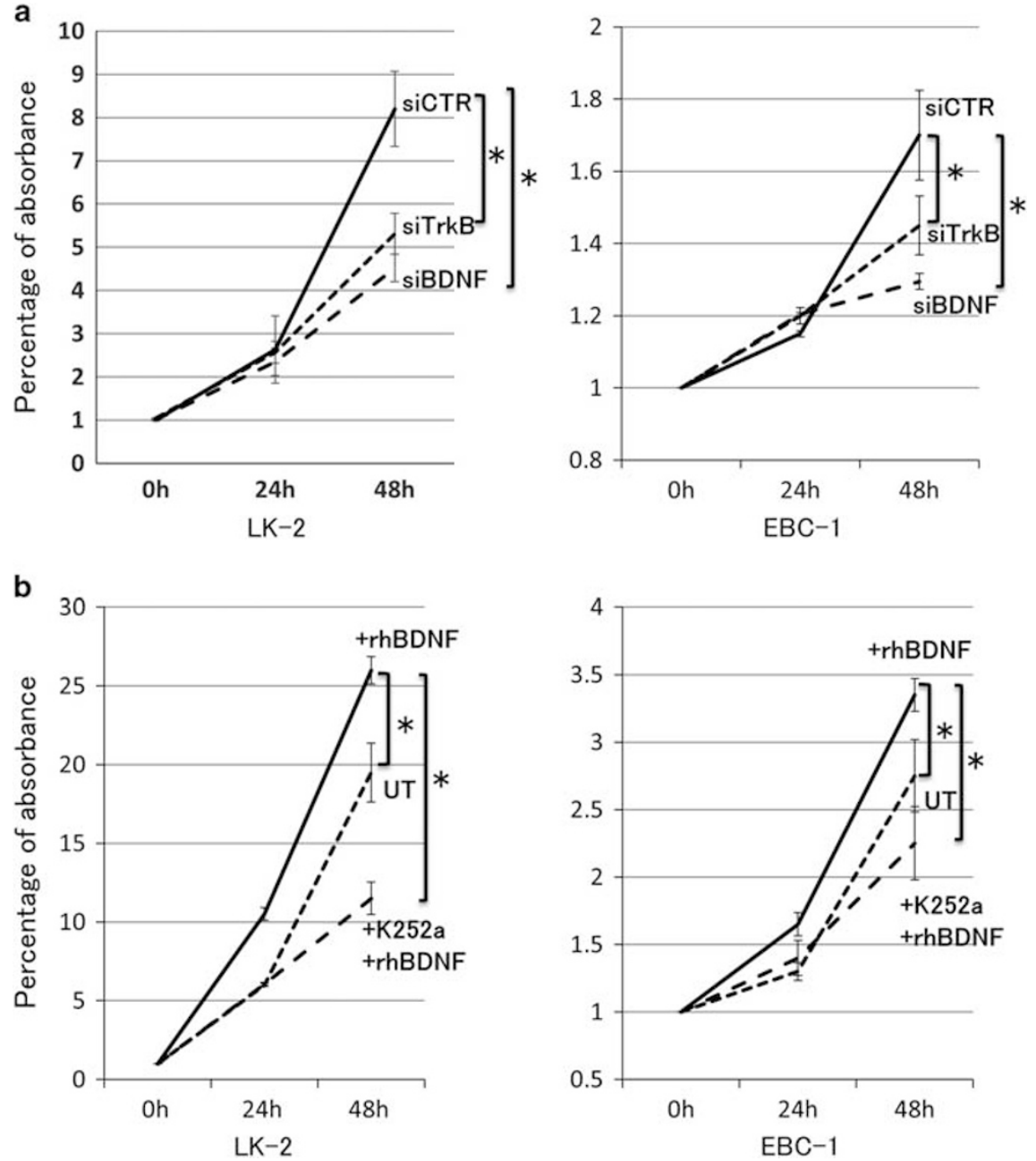

Figure 4 The BDNF/TrkB pathway is involved in proliferation activity. (a) LK-2 and EBC-1 cells transfected with the TrkB-siRNA or BDNF-siRNA showed low levels of proliferation compared to those transfected with Control-siRNA. (b) LK-2 and EBC-1 cells incubated with rhBDNF (10 ng/ml) showed enhanced proliferation activity, and K252a $(50 \mathrm{nM})$ abrogated this activity. ${ }^{*} P<0.05,{ }^{*} P<0.01$. UT, Untreated.

Figure 3 The Trk inhibitor K252a inhibited the invasion and migration activity of lung SCC cells with endogenous TrkB and BDNF expressions. (a) Human lung SCC cell lines (LC-1 sq, LK-2, RERF-LC-Al, and EBC-1) were subjected to a western blot analysis. The LK-2 and EBC-1 cells showed endogenous TrkB and BDNF expression. The LK-2 and EBC-1 cells, incubated with K252a (50 and $100 \mathrm{nM}$ ), showed decreased numbers of (b) invasion and (c) migration cells. Error bars: s.d. Exogenous rhBDNF enhanced the cells' invasion and migration abilities, and K252a abrogated these abilities. LK-2 and EBC-1 cells incubated with rhBDNF (10 and $100 \mathrm{ng} / \mathrm{ml}$ ) showed enhanced (d) invasion and (e) migration abilities. LK-2 and EBC-1 cells incubated with both rhBDNF $(10 \mathrm{ng} / \mathrm{nl})$ and $\mathrm{K} 252 \mathrm{a}(50 \mathrm{nM})$ showed abrogated (f) invasion and (g) migration abilities. Error bars: $\mathrm{s} . \mathrm{d}$. ${ }^{*} P<0.05,{ }^{* *} P<0.01,{ }^{5} P<0.005$. 
numbers of invasion and migration cells irrespective of being transfected with BDNF-siRNA or TrkB-siRNA or ControlsiRNA (Supplementary Figure S1E).

\section{The Administration of Recombinant Human BDNF Enhanced the Invasion and Migration Activities of Lung SCC Cells, and K252a Abrogated These Activities}

After the administration of 10 or $100 \mathrm{ng} / \mathrm{ml} \mathrm{rhBDNF}$ to the respective culture media of LK-2 and EBC-1 cells, the numbers of invading cells and migrating cells were significantly increased (Figure 3d and e), indicating that rhBDNF enhanced the invasion and migration activities.

After the administration of $50 \mathrm{nM} \mathrm{K} 252 \mathrm{a}$ and $10 \mathrm{ng} / \mathrm{ml}$ rhBDNF to the respective culture media of LK-2 and EBC-1 cells, the numbers of invading cells and migrating cells were significantly decreased compared to those after the administration of rhBDNF alone (Figure $3 \mathrm{f}$ and $\mathrm{g}$ ), indicating that $\mathrm{K} 252 \mathrm{a}$ abrogated the enhancement of the invasion and migration activities.

\section{The Inhibition of BDNF/TrkB Signaling by siRNA Suppressed the Anchorage-Dependent Proliferation Activity of Lung SCC Cells}

To assess the effect of the BDNF/TrkB signaling pathway on proliferation, we performed an anchorage-dependent proliferation assay. The LK-2 and EBC-1 cells transfected with TrkB-siRNA or BDNF-siRNA showed significantly low levels of absorbance compared to those transfected with ControlsiRNA (Figure 4a), indicating that anchorage-dependent proliferation activity was suppressed.

\section{The Trk Inhibitor K252a Can Abrogate the Anchorage- Dependent Proliferation Activity of Lung SCC Cells}

The cells incubated with $10 \mathrm{ng} / \mathrm{ml} \mathrm{rhBDNF}$ showed significantly higher levels of absorbance compared to the cells incubated without rhBDNF, indicating that anchoragedependent proliferation activity was enhanced by rhBDNF.

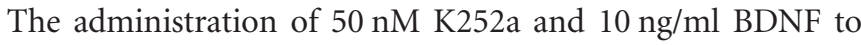
the respective cultured media of LK-2 and EBC-1 cells a

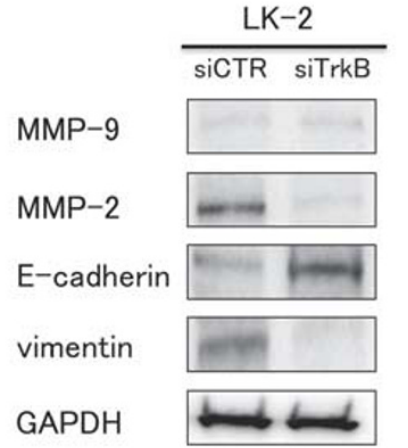

$\mathrm{EBC}-1$
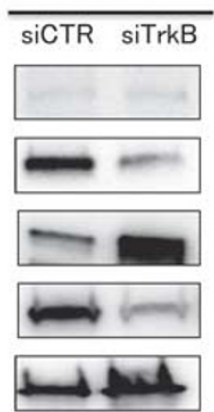

b
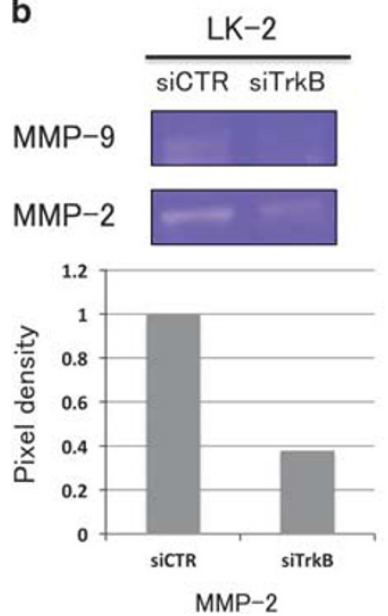
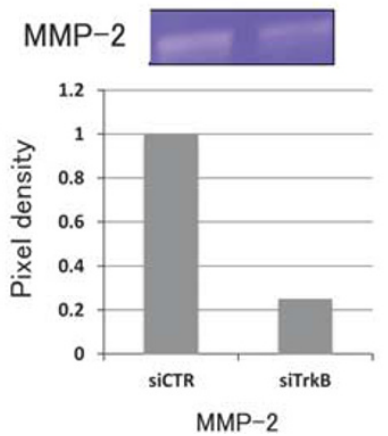

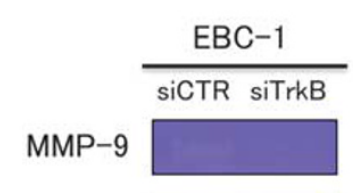

C

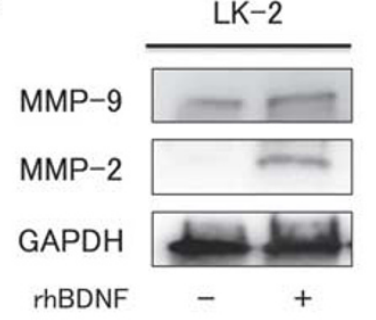

d
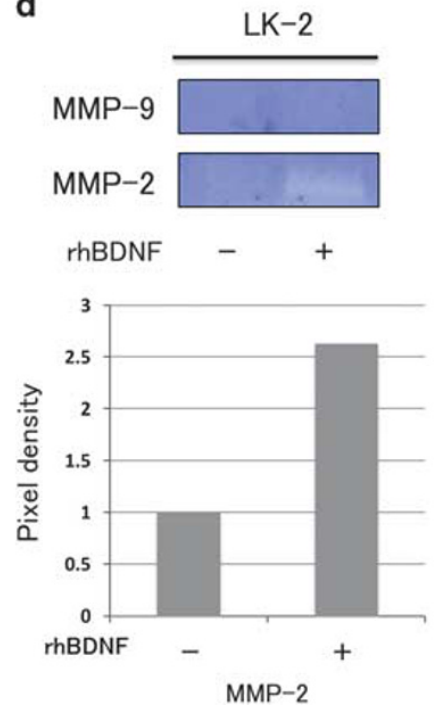
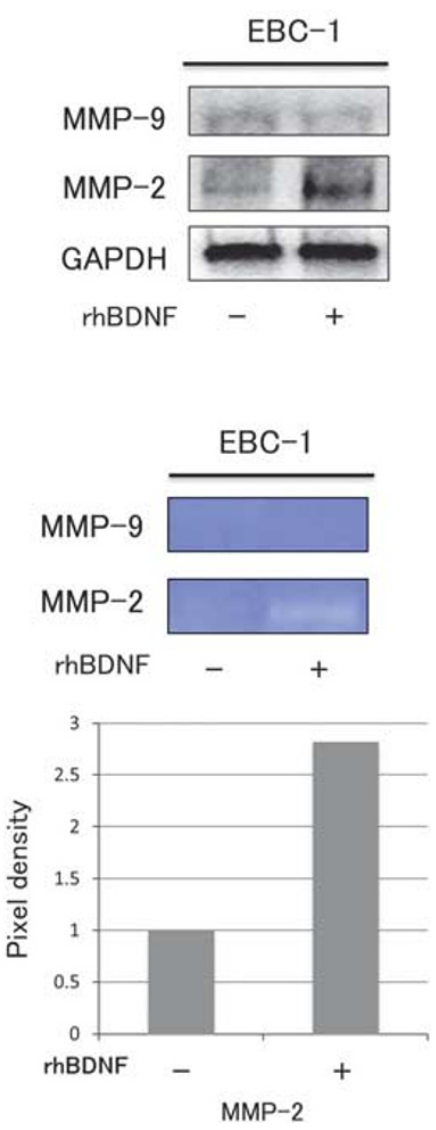

Figure $\mathbf{5}$ TrkB is involved in the EMT and the expression/activation of MMP-2. Western blotting showed (a) decreased protein levels of MMP-2 and vimentin and an increased protein level of E-cadherin in cells transfected with TrkB-siRNA compared to those transfected with control-siRNA. Gelatin zymography showed (b) decreased activity of MMP-2 in the medium of the cells transfected with TrkB-siRNA, whereas no difference was detected in the activity of MMP-9. The panels show the densitometry analysis results. Western blotting also showed (c) increased protein levels of MMP-2 in cells administrated with rhBDNF compared to those without rhBDNF, whereas no difference was detected in the expression of MMP-9. Gelatin zymography also showed (d) increased activity of MMP-2 in the medium of the cells administrated with rhBDNF, whereas no difference was detected in the activity of MMP-9. The panels show the densitometry analysis results. CTR, control. 
resulted in lower levels of absorbance than the administration of rhBDNF alone, indicating that K252a abrogated the enhanced anchorage-dependent proliferation activity by rhBDNF (Figure $4 \mathrm{~b}$ ). The RERF-LC-AI cell, without endogenous TrkB expression, showed no significant changes in absorbance irrespective of being treated with $50 \mathrm{nM} \mathrm{K252a}$ (Supplementary Figure S3).

\section{The Western Blot Analysis Showed Increased E-cadherin and Decreased Vimentin Expression of the Cells Transfected With TrkB-siRNA}

To clarify the underlying mechanisms mediating invasion by $\mathrm{BDNF} / \mathrm{TrkB}$ signaling, we investigated whether $\operatorname{TrkB}$ is involved in the epithelial-mesenchymal transition (EMT) in lung SCC cells. In the Western blot analysis, each cell line transfected with TrkB-siRNA showed the increased protein expression of E-cadherin and decreased vimentin compared to the cells transfected with Control-siRNA (Figure 5a), indicating that TrkB promoted the EMT of lung SCC cells.

\section{The Downregulation of TrkB by siRNA Inhibited the MMP-2 Expression and Gelatin-Degrading Activity of MMP-2}

To clarify other mechanisms mediating invasion, we investigated whether TrkB influences members of the matrix metalloproteinase family, ie, MMP-2 and MMP-9. Western blotting showed lower MMP-2 expression of the cells transfected with TrkB-siRNA compared to the cells transfected with control-siRNA, whereas the expression of MMP-9 was not significantly different (Figure 5a).

Gelatin zymography demonstrated reduced gelatin degradation by MMP-2 in the culture media of LK- 2 and EBC- 1 cells transfected with TrkB-siRNA compared to those transfected with control-siRNA. No significant differences of gelatin degradation by MMP-9 in the two media were detected (Figure 5b).

\section{Administration of Recombinant Human BDNF Enhanced MMP-2 Expression and the Gelatin-Degrading Activity of MMP-2}

To clarify whether MMP functions were enhanced by the incubation with rhBDNF, we performed a western blot analysis and gelatin zymography. The western blotting showed higher MMP-2 expression of the cells administered rhBDNF compared to those without rhBDNF, whereas the expression of MMP-9 was not significantly different (Figure 5c).

Gelatin zymography demonstrated that gelatin degradation was induced by MMP-2 in the culture media of both cells administered rhBDNF compared to those without rhBDNF, whereas no significant difference of gelatin degradation by MMP-9 in the two media was detected (Figure 5d). These results indicated that $\mathrm{TrkB}$ enhanced the expression of MMP-2 and the gelatin-degrading activity of MMP-2 in lung SCC cells.

\section{DISCUSSION}

This study was designed to analyze the biological significance of Trk family members in lung SCC and to investigate whether the Trk signal pathway could be a therapeutic target. Many studies have indicated that Trk is oncogenic in several malignant tumors, including lung cancer, ${ }^{18-44}$ and some of those studies demonstrated the BDNF and TrkB expressions in lung cancer. ${ }^{39-44}$ Similar to our results, high TrkB expression in NSCLC was reported to be correlated with lymph node metastasis ${ }^{40}$ and vascular invasion. ${ }^{41}$ In the present study, we focused on squamous cell carcinoma histology, and our findings demonstrated the correlation between high TrkB expression and lymph node metastasis/ vascular invasion. Okamura et al. ${ }^{41}$ also observed that $\operatorname{TrkB}$ expression was correlated with unfavorable prognosis in NSCLC including lung SCC, in line with our results. Other researchers also reported that $\operatorname{TrkB}$ expression was correlated with lymph node metastasis and/or advanced stage in lung adenocarcinoma, ${ }^{40}$ colon cancer ${ }^{31,33}$ and gastric cancer. ${ }^{29}$

However, we should point out that there is a controversial study demonstrating that lung SCC patients with high TrkB expression showed favorable prognoses. ${ }^{42}$ A possible explanation for this discrepancy is that a different method was used to evaluate the expression level of TrkB immunohistochemically in that study, as a standard method had not yet been established. To the best of our knowledge, that study ${ }^{42}$ was the only study demonstrating that $\mathrm{TrkB}$ expression is an indicator of favorable prognosis in malignant tumors. Again, our results are in line with the many other studies demonstrating that $\operatorname{TrkB}$ is an indicator of poor prognosis in malignant tumors. ${ }^{23-36,39-41}$ Further analyses are necessary to clarify the appropriateness of the exceptional results regarding TrkB's role, using similar technical processes.

With respect to TrkC, our present analysis revealed that all of the patients with high TrkC expression were under stage II, and their overall survival rate was higher than that of the TrkC-low expression patients. Our findings demonstrated that $\mathrm{TrkC}$ is a marker of good prognosis in lung SCC, as was also indicated in malignant tumors such as medulloblastoma ${ }^{37}$ and colon cancer. ${ }^{38}$ We observed no correlations between TrkA expression and clinicopathological factors in the present investigation.

On the basis of our observation that $\operatorname{TrkB}$ expression is an indicator of poor prognosis in lung SCC samples, we investigated the biological effects of the BDNF/TrkB signaling pathway on lung SCC cells in vitro. The results demonstrated that the inhibition of the BDNF/TrkB pathway by K252a or siRNA diminished the invasion and migration of lung SCC cells. Similar results were reported in other types of lung cancer such as adenocarcinoma cells, ${ }^{40}$ LCNEC cells, ${ }^{43}$ colon cancer cells, ${ }^{33}$ and ovarian cancer cells. ${ }^{26}$ Zhang et al. showed that BDNF facilitates the tumorgenesis and progression of NSCLC (SCC and adenocarcinoma), ${ }^{47}$ in line with our results. In this context, we have systematically investigated the $\mathrm{BDNF} / \mathrm{TrkB}$ signal by inhibiting both BDNF (ligand) and 
TrkB (receptor), and substantiated Zhang's results. Furthermore, our study is focused on SCC.

In the present study, there were no significant differences in the numbers of invading/migrating cells between the uses

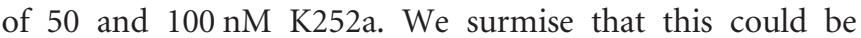
explained by a saturation of the TrkB.

Our present findings also demonstrated that the administration of rhBDNF enhanced invasion and migration, and K252a abrogated these activities. Similar results were reported in LCNEC $^{43}$ and gastric cancer ${ }^{28}$ cells. In our study, however, the numbers of invading cells and migrating cells administered the combination of rhBDNF and K252a were somewhat greater than those obtained with K252a alone. In this context, we speculate that it is possible that rhBDNF can promote cell invasion/migration via a pathway other than a Trk pathway, and that K252a could not inhibit this alternate signal pathway.

These results suggest that an elevated BDNF/TrkB signaling pathway generally enhances the invasion and migration activities in various types of malignant tumors including lung SCC (Figure 1).

The EMT, usually expressed by E-cadherin downregulation and vimentin upregulation, is an important step in the invasion process. ${ }^{48}$ It was reported that $\mathrm{BDNF} / \mathrm{TrkB}$ induces the EMT in gastric cancer cells, ${ }^{28}$ colon cancer cells, ${ }^{33}$ oral squamous carcinoma cells, ${ }^{34}$ and head and neck squamous cell carcinoma cells. ${ }^{35}$ Our present findings demonstrated an increased level of E-cadherin protein expression and a decreased level of vimentin protein expression in lung SCC cells transfected with TrkB-siRNA. Our results suggest that TrkB signal induced the EMT of lung SCC cells, as demonstrated in other malignant tumors. ${ }^{28,33-35}$

The degradation of the extracellular matrix (ECM) and basement membrane by MMP, especially MMP-2 and MMP-9, is also an important component of the invasion process. ${ }^{49}$ Odate et al. reported that the BDNF/TrkB signal promotes the expression of both MMP-2 and MMP-9 in LCNEC cells, ${ }^{43}$ and Kupferman et al. showed that the BDNF/ TrkB signal promotes the expression and activation of MMP-9, but not MMP-2. ${ }^{35}$ In our present investigation, TrkB induced the expression and activation of MMP-2 but not MMP-9 in lung SCC cells. In this context, we surmise that the subtypes of MMP mediating invasion may differ among tumor types. We speculate that TrkB is involved in invasion activity through increasing migration activity, leading to the EMT and the expression and activation of MMP-2 but not MMP-9 in lung SCC cells (Figure 1).

In addition to the invasion process, it was demonstrated that the BDNF/TrkB pathway was involved in the cell proliferation of various types of malignant cells, including hepatocellular carcinoma, $^{24}$ gastric cancer, $^{28}$ colon cancer, ${ }^{31,33}$ neuroblastoma, ${ }^{36}$ and lung adenocarcinoma. ${ }^{44}$ Those results are consistent with ours, demonstrating that the BDNF/TrkB pathway is involved in the proliferation of lung SCC cells (Figure 1). However, Odate et al. reported that the BDNF/TrkB pathway was not involved in the proliferation of LCNEC cells. ${ }^{43}$ The effect of the BDNF/TrkB pathway on cell proliferation activity may differ among tumor types.

Our present findings demonstrated that the downregulation of BDNF by siRNA inhibited the BDNF/TrkB signal pathway, resulting in decreased invasion, migration, and proliferation activities. Our findings also demonstrated that the administration of rhBDNF enhanced the BDNF/TrkB signal pathway, resulting in increased invasion, migration and proliferation activities. These results suggest that endogenous BDNF produced by lung SCC cells functions in an autocrine and/or paracrine manner. On the basis of the results of our in vitro assay, the BDNF/TrkB signaling pathway amplified by an autocrine/paracrine effect of BDNF production may contribute to the aggressive behavior of lung SCC.

This is the first study to demonstrate by using a Trk inhibitor that the inhibition of TrkB suppresses the migration, invasion and proliferation activities of lung SCC. These results may pave the way for a target therapy for lung SCC. We speculate that a Trk inhibitor could become eventually one of the choices of therapy for lung SCC patients, since some Trk inhibitors such as Entrectinib and LOXO-101 are already being investigated in phase 2, and Khotskaya $\mathrm{YB}$ et al. reported that these inhibitors demonstrated clinical activity among patients whose tumors harbor an NTRK gene fusion. ${ }^{50}$ The relationship between overexpression of NTRK gene alterations should be investigated intensively in the future.

In conclusion, the BDNF/TrkB signaling pathway contributes to the aggressive behavior of lung SCC by promoting cell invasion and proliferation activities, and this pathway may be a new therapeutic target for lung SCC (Figure 1).

Supplementary Information accompanies the paper on the Laboratory Investigation website (http://www.laboratoryinvestigation.org)

\section{ACKNOWLEDGMENTS}

We thank Manabu Yasuda (Department of Thoracic Surgery, Shinkokura Hospital) and Tomoyuki Hida (Department of Clinical Radiology, Graduate School of Medical Sciences, Kyushu University) for their kind support.

\section{DISCLOSURE/CONFLICT OF INTEREST}

The authors declare no conflict of interest.

1. Siegel RL, Miller KD, Jemal A. Cancer statistics. CA Cancer J Clin 2016;66:7-30.

2. Travis WD. Pathology of lung cancer. Clin Chest Med 2011;32:669-692.

3. Lynch TJ, Bell DW, Sordella R, et al. Activating mutations in the epidermal growth factor receptor underlying responsiveness of nonsmall-cell lung cancer to gefitinib. N Engl J Med 2004;350:2129-2139.

4. Mok TS, Wu YL, Thongprasert S, et al. Gefitinib or carboplatin-paclitaxel in pulmonary adenocarcinoma. N Engl J Med 2009;361:947-957.

5. Maemondo M, Inoue A, Kobayashi K, et al. Gefitinib or chemotherapy for non-small-cell lung cancer with mutated EGFR. N Engl J Med 2010;362:2380-2388.

6. Petrelli F, Borgonovo $\mathrm{K}$, Cabiddu $\mathrm{M}$, et al. Efficacy of EGFR tyrosine kinase inhibitors in patients with EGFR-mutated non-small-cell lung cancer: a meta-analysis of 13 randomized trials. Clin Lung Cancer 2012;13:107-114. 
7. Solomon BJ, Mok T, Kim DW, et al. First-line crizotinib versus chemotherapy in ALK-positive lung cancer. N Engl J Med 2014;371: 2167-2177.

8. Camidge DR, Doebele RC. Treating ALK-positive lung cancer-early successes and future challenges. Nat Rev Clin Oncol 2012;9:268-277.

9. Thatcher N, Chang A, Parikh P, et al. Gefitinib plus best supportive care in previously treated patients with refractory advanced non-small-cell lung cancer: results from a randomised, placebo-controlled, multicentre study (Iressa Survival Evaluation in Lung Cancer). Lancet 2005;366:1527-1537.

10. Ameratunga M, Pavlakis N, Gebski V, et al. Epidermal growth factor receptor-tyrosine kinase inhibitors in advanced squamous cell carcinoma of the lung: a meta-analysis. Asia Pac J Clin Oncol 2014;10:273-278.

11. Achille M, Gallegos-Ruiz M, Giaccone $G$, et al. Response to erlotinib in first-line treatment of non-small-cell lung cancer in a white male smoker with squamous-cell histology. Clin Lung Cancer 2006;8:214-216.

12. Fang $W$, Zhang J, Liang $W$, et al. Efficacy of epidermal growth factor receptor-tyrosine kinase inhibitors for Chinese patients with squamous cell carcinoma of lung harboring EGFR mutation. J Thorac Dis 2013;5: 585-592.

13. Hata A, Katakami N, Yoshioka $\mathrm{H}$, et al. How sensitive are epidermal growth factor receptor-tyrosine kinase inhibitors for squamous cell carcinoma of the lung harboring EGFR gene-sensitive mutations? J Thorac Oncol 2013;8:89-95.

14. Chao MV, Bothwell MA, Ross $\mathrm{AH}$, et al. Gene transfer and molecular cloning of the human NGF receptor. Science 1986;232:518-521.

15. Hantzopoulos PA, Suri C, Glass DJ, et al. The low affinity NGF receptor, p75, can collaborate with each of the Trks to potentiate functional responses to the neurotrophins. Neuron 1994;13:187-201.

16. Klein R, Parada LF, Coulier F, et al. trkB, a novel tyrosine protein kinase receptor expressed during mouse neural development. EMBO J 1989;8: 3701-3709.

17. Klein R, Smeyne RJ, Wurst W, et al. Targeted disruption of the trkB neurotrophin receptor gene results in nervous system lesions and neonatal death. Cell 1993;75:113-122.

18. McGregor LM, McCune BK, Graff JR, et al. Roles of trk family neurotrophin receptors in medullary thyroid carcinoma development and progression. Proc Natl Acad Sci USA 1999;96:4540-4545.

19. Lagadec C, Meignan S, Adriaenssens $E$, et al. TrkA overexpression enhances growth and metastasis of breast cancer cells. Oncogene 2009;28:1960-1970.

20. Davidson $B$, Reich $R$, Lazarovici $P$, et al. Expression and activation of the nerve growth factor receptor TrkA in serous ovarian carcinoma. Clin Cancer Res 2003;9:2248-2259.

21. Gao Q, Chen CF, Dong Q, et al. Elevated TrkA receptor expression is associated with all-trans retinoic acid-induced neuroblastoma differentiation. Genet Mol Res 2015;14:13195-13202.

22. Anagnostopoulou V, Pediaditakis I, Alkahtani S, et al. Differential effects of dehydroepiandrosterone and testosterone in prostate and colon cancer cell apoptosis: the role of nerve growth factor (NGF) receptors. Endocrinology 2013;154:2446-2456.

23. Sclabas GM, Fujioka S, Schmidt C, et al. Overexpression of tropomysinrelated kinase $B$ in metastatic human pancreatic cancer cells. Clin Cancer Res 2005;11(2 Pt 1):440-449.

24. Yang ZF, Ho DW, Lam CT, et al. Identification of brain-derived neurotrophic factor as a novel functional protein in hepatocellular carcinoma. Cancer Res 2005;65:219-225.

25. Satoh F, Mimata H, Nomura T, et al. Autocrine expression of neurotrophins and their receptors in prostate cancer. Int J Urol 2001;8:S28-S34.

26. $\mathrm{Yu} X$, Liu L, Cai B, et al. Suppression of anoikis by the neurotrophic receptor TrkB in human ovarian cancer. Cancer Sci 2008;99:543-552.

27. Eggert A, Grotzer MA, Ikegaki N, et al. Expression of the neurotrophin receptor TrkB is associated with unfavorable outcome in Wilms' tumor. J Clin Oncol 2001;19:689-696.

28. Okugawa $Y$, Tanaka K, Inoue $Y$, et al. Brain-derived neurotrophic factor/ tropomyosin-related kinase $\mathrm{B}$ pathway in gastric cancer. $\mathrm{Br} \mathrm{J}$ Cancer 2013;108:121-130.
29. Tanaka K, Mohri Y, Nishioka J, et al. Neurotrophic receptor, tropomyosin-related kinase $B$ as an independent prognostic marker in gastric cancer patients. J Surg Oncol 2009;99:307-310.

30. Patani N, Jiang WG, Mokbel K. Brain-derived neurotrophic factor expression predicts adverse pathological \& clinical outcomes in human breast cancer. Cancer Cell Int 2011;11:23.

31. Yu Y, Zhang S, Wang $X$, et al. Overexpression of TrkB promotes the progression of colon cancer. APMIS 2010;118:188-195.

32. Sasahira T, Ueda N, Kurihara M, et al. Tropomyosin receptor kinases B and $\mathrm{C}$ are tumor progressive and metastatic marker in colorectal carcinoma. Hum Pathol 2013;44:1098-1106.

33. Fujikawa $\mathrm{H}$, Tanaka $\mathrm{K}$, Toiyama $\mathrm{Y}$, et al. High TrkB expression levels are associated with poor prognosis and EMT induction in colorectal cancer cells. J Gastroenterol 2012;47:775-784.

34. Dudas J, Bitsche M, Schartinger V, et al. Fibroblasts produce brainderived neurotrophic factor and induce mesenchymal transition of oral tumor cells. Oral Oncol 2011;47:98-103.

35. Kupferman ME, Jiffar T, El-Naggar A, et al. TrkB induces EMT and has a key role in invasion of head and neck squamous cell carcinoma. Oncogene 2010;29:2047-2059.

36. Nakagawara A, Azar CG, Scavarda NJ, et al. Expression and function of TRK-B and BDNF in human neuroblastomas. Mol Cell Biol 1994;14: 759-767.

37. Grotzer MA, Janss AJ, Phillips PC, et al. Neurotrophin receptor TrkC predicts good clinical outcome in medulloblastoma and other primitive neuroectodermal brain tumors. Klin Padiatr 2000;212: 196-199.

38. Genevois AL, Ichim G, Coissieux MM, et al. Dependence receptor TrkC is a putative colon cancer tumor suppressor. Proc Natl Acad Sci USA 2013;110:3017-3022.

39. Ricci A, Greco S, Mariotta S, et al. Neurotrophins and neurotrophin receptors in human lung cancer. Am J Respir Cell Mol Biol 2001;25: 439-446.

40. Zhang S, Guo D, Luo W, et al. TrkB is highly expressed in NSCLC and mediates BDNF-induced the activation of Pyk2 signaling and the invasion of A549 cells. BMC Cancer 2010;10:43.

41. Okamura K, Harada T, Wang $S$, et al. Expression of TrkB and BDNF is associated with poor prognosis in non-small cell lung cancer. Lung Cancer 2012;78:100-106.

42. Terry J, De Luca A, Leung S, et al. Immunohistochemical expression of neurotrophic tyrosine kinase receptors 1 and 2 in lung carcinoma: potential discriminators between squamous and nonsquamous subtypes. Arch Pathol Lab Med 2011;135:433-439.

43. Odate $\mathrm{S}$, Nakamura $\mathrm{K}$, Onishi $\mathrm{H}$, et al. TrkB/BDNF signaling pathway is a potential therapeutic target for pulmonary large cell neuroendocrine carcinoma. Lung Cancer 2013;79:205-214.

44. Perez-Pinera P, Hernandez T, Garcia-Suarez O, et al. The Trk tyrosine kinase inhibitor K252a regulates growth of lung adenocarcinomas. Mol Cell Biochem 2007;295:19-26.

45. Travis WD, Brambilla E, Nicholson AG, et al. The 2015 World Health Organization Classification of lung tumors: impact of genetic, clinical and radiologic advances since the 2004 classification. J Thorac Oncol 2015;10:1243-1260.

46. Greene FL, Sobin LH. A worldwide approach to the TNM staging system: collaborative efforts of the AJCC and UICC. J Surg Oncol 2009;99:269-272.

47. Zhang SY, Hui LP, Li CY, et al. More expression of BDNF associates with lung squamous cell carcinoma and is critical to the proliferation and invasion of lung cancer cells. BMC Cancer 2016;16:171.

48. Hay ED. An overview of epithelio-mesenchymal transformation. Acta Anat (Basel) 1995;154:8-20.

49. Folgueras AR, Pendas AM, Sanchez LM, et al. Matrix metalloproteinases in cancer: from new functions to improved inhibition strategies. Int J Dev Biol 2004;48:411-424.

50. Khotskaya YB, Holla VR, Farago AF et al. Targeting TRK family proteins in cancer. Pharmacol Ther 2017. e-pub ahead of print 4 February 2017; doi: 10.1016/j.pharmthera.2017.02.006. 